\title{
MEASUREMENT OF THE FREE CARRIER FARADAY EFFECT IN $n$-TYPE GERMANIUM AT MICROWAVE FREQUENCIES
}

\author{
by A. BOUWKNEGT and J. VOLGER
}

Fysisch Laboratorium der Rijksuniversiteit, Utrecht, Nederland

\section{Synopsis}

Measurements of the Faraday rotation and ellipticity on $n$-type (001) oriented germanium crystals at $24.9 \mathrm{GHz}$ and at room temperature have been performed, using a crossed wave guide method. The influences of multiple reflections and of the surfaces on the Faraday effect have been analysed. These effects can be eliminated in a simple way. The rotation and ellipticity for the samples used were calculated, using the multi valley model for $n$-type germanium. The agreement between theory and experiment was found to be good.

1. Introduction. The effect of a magnetic field on a linearly polarized electromagnetic wave, propagating through a medium into the direction of the magnetic field, is known as the Faraday effect. At microwave frequencies the free carriers in a semiconductor, interacting with the electromagnetic field in the presence of the external magnetic field, cause a transformation of a linearly polarized wave into an elliptically polarized one. The major axis of the ellips is rotated with respect to the inilial polarization.

Faraday rotation at microwave frequencies was observed by Suhl and Pearson ${ }^{1}$ ) and Rau and Caspari $\left.{ }^{2}\right)$. Measurements on both rotation and ellipticity were reported by Furdyna and Broersma ${ }^{3}$ ). The theory of the free carrier Faraday effect was given by Stephen and Lidiard ${ }^{4}$ ). Donovan and Webster ${ }^{5}$ ) and Furdyna and Brodwin ${ }^{6}$ ) extended the theory by introducing an energy dependent relaxation time, the latter authors treated the ellipticity. The theory of the Faraday effect in anisotropic media was given by Donovan and $\left.\left(\operatorname{cbstcr}^{7}\right)^{8}\right)$.

In the microwave frequency region the inverse relaxation time can easily be of the same order of magnitude as the frequency of the electromagnetic field. In this important region information about the scattering mechanisms can be obtained from measurements of the ellipticity ${ }^{6}$ ) or from a combination of the Faraday rotation and ellipticity for weak magnetic fields with the propagation constants for these fields. The latter was recently pointed out by $\left.\mathrm{Champlin}^{9}\right)$. However it is necessary that the measurements are 
carricd out with good accuracy. Even after the work of Furdyna and Broersma ${ }^{3}$ ) additional experimental information would, therefore, be valuable.

It is the purpose of this paper to discuss the merits of a crossed wave guide measuring technique, hitherto not used as far as the present authors know. Measurements on $n$-type germanium at $24.9 \mathrm{GHz}$ will be reported. In discussing these measurements multiple reflections will be taken into account and a comparison will be made with theoretically expected values.

2. Method. The method of measurement used is schematically drawn in fig. 1. The vital part is a crossed wave guide construction, a somewhat modified version of the wave guide coupler proposed by Hambleton and Gärtner $\left.{ }^{10}\right)$. This coupler is drawn in fig. $1 a$, it can be put either in the crossed or in the parallel position. In the narrow wall of the two wave guides a coupling hole is carefully machined. It is usually square with its side as large as the internal width $a$ of the guide. The width $b$ is slightly larger than half the free space wave length of the electromagnetic field used. Between the two halfs of the coupler the planparallel germanium crystal is tightly mounted, its diameter is much larger than the hole dimensions. The two irises are adjusted as accurately as possible, facing each other. The primary guide is closed at one side with a plunger, the secondary one with a matched load.

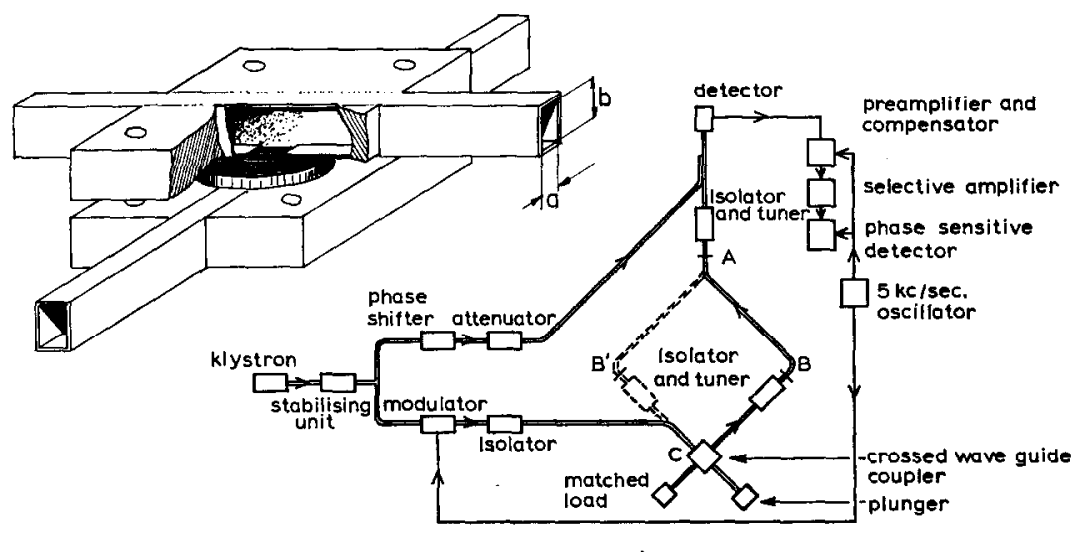

a

b

Fig. 1. a) Drawing of the wave guide coupler.

b) Simplified scheme of measuring circuit.

The electromagnetic field from the primary guide penetrates the crystal and can thus be radiated into the secondary one. However, only the electric field component perpendicular to the direction of the secondary wave guide couples with the field in it. The energy radiated towards 
the detector is therefore zero when the two guides are perpendicular to each other and no external magnetic field is present. If the field is present, the signal detected in the secondary guide is entirely due to the Faraday rotation. Phase and amplitude of the field radiated from the germanium crystal are measured in two directions, by adjusting the secondary guide successively perpendicular and parallel to the primary one. From these measured values the specific rotation and ellipticity due to the Faraday effect can be derived.

The phase and amplitude are measured in the bridge sketched in fig. $1 b$. The method has partly been described by Schafer $\left.{ }^{11}\right)$. The microwave energy is carefully frequency- and energy- stabilized before entering the bridge. In the bridge arm containing the coupler a $5 \mathrm{kHz}$ amplitude modulator is inserted. The energy, emitted by the semiconductor crystal, passes either from $C$ via $B$ to $A$ (transverse position), or from $C$ via $B^{\prime}$ to $A$ (parallel position). In view of the phase measurements the electrical path $C-B-A$ must be exactly equal to $C-B^{\prime}-A$. To avoid standing waves between $A$ and $B$ or $A$ and $B^{\prime}$ two isolators with tuners were incorporated. The coupler must be machined with high precision, typical tolerances for the one used were $0.03 \mathrm{~mm}$.

Four measurements yield the phase and amplitude of the emitted electric field with high accuracy. Additionally, these measurements are performed with positive and negative sign of the applied magnetic field $B$. In this way almost all the perturbing effects can be eliminated, assuming they are small, and even in $B$. To mention some: a small phase modulation by the modulator, mode conversion in the coupler, leakages in the coupler in the transverse position or deviations from the perpendicular position of the guides. The amplitude detection is linear, when the behaviour of the detection crystal is not too far from quadratic. The linearity of the system can easily be checked. The $5 \mathrm{kHz}$ signal was measured with a compensation method.

The reproducibility of the phase measurements is about $0.5^{\circ}$, the amplitude measurements are reproducible within $2 \%$.

Since an exact theory of the field distribution in and near the sample is not available in the case of the configuration used, a number of simplifying assumptions are made for the discussion of the derivation of specific Faraday rotation and ellipticity from the observed quantities. Firstly it has been taken that the field of the $\mathrm{TE}_{\mathbf{0 1}}$ mode in the primary rectangular wave guide is not too much disturbed by the presence of the semiconductor crystal. This approximation is the better the higher the conductivity of the crystal and the smaller the iris. The plunger is adjusted for maximum microwave field at the iris to occur, i.e. the incident field is assumed to be an undisturbed complete standing wave.

Together with this first assumption it is secondly assumed that the field inside the crystal can be approximated by a symmetrical superposition of 
damped plane waves, which propagate nearly parallel to the external magnetic field. Of course other modes, propagating at larger angles to the magnetic field, are excited as well. However, their contribution to the emitted electric field should be of less importance, due to the high refractive index (about 4!) and the absorption of the crystal. Consequently it is expected that they will not disturb the total Faraday rotation and ellipticity much. The field not emitted directly from the primary iris to the secondary one is totally absorbed in the crystal.

A plane wave, propagating at an angle with the magnelic field, causes a rotation and an ellipticity that differs slightly from that, caused by an exactly parallel propagating wave. An estimation showed that the deviations are of second order as long as the angle is less than, say, 10 to 15 degrees. In view of the foregoing considerations the plane wave treatment of the Faraday effect seems reasonable in the case of the crossed wave guide problem.

Multiple reflections and surface effects can influence microwave measurements of the Faraday effect considerably, especially the cllipticity. Rccently these effects were in principle analysed for cylindrical guides by Champlin ${ }^{8}$ ), using well known transmission formulae for a thin sheet of conducting material. From an experimental point of view the following is of importance:

The complex amplitude transmission coefficient $T$ for a sheet of conducting medium in free space is, for any electromagnetic plane wave, that is incident upon the semi infinite slab, perpendicular to the surface:

$$
T=T_{1} T_{2} \mathrm{e}^{i \gamma d}\left[\frac{1}{1-R_{1} R_{2} \mathrm{e}^{2 i \gamma d}}\right]=T_{1} T_{2} \mathrm{e}^{i \gamma d} M
$$

with $T_{1}$ and $T_{2}$ the transmission coefficients of the first and second surface respectively, $R_{1}$ and $R_{2}$ the internal reflection coefficients, $d$ the thickness of the slab and $\gamma$ the propagation constant of the material. The last factor in the equations is due to internal reflections in the slab. Of course $T_{1}$, $T_{2}, R_{1}$ and $R_{2}$ are complex. If one writes: $T_{1,2}=\mathrm{e}^{-t_{1,2}} \mathrm{e}^{i \varphi_{1,2}}, M=\mathrm{e}^{-\delta} \mathrm{e}^{i \Delta}$ and $\gamma=\alpha+i \beta$, and denotes the right- and left handed circular polarization by the subscriptions,+- , the rotation is:

$$
\Theta=-\left[\frac{\alpha_{+}-\alpha_{-}}{2} d+\frac{\left(\varphi_{1}+\varphi_{2}\right)_{+}-\left(\varphi_{1}+\varphi_{2}\right)_{-}}{2}+\frac{\Delta_{+}-\Delta_{-}}{2}\right]
$$

and the ellipticity:

$$
E=\tanh .\left[\frac{\beta_{+}-\beta_{-}}{2} d+\frac{\left(t_{1}+t_{2}\right)_{+}-\left(t_{1}+t_{2}\right)_{-}}{2}+\frac{\delta_{+}-\delta_{-}}{2}\right] .
$$

Thus both effects can be split up into a) the intrinsic effect, proportional to $d$, b) an effect due to the surfaces, this term is closely related to the 
magneto-optical Kerr effect, and independent of $d$, and c) an effect due to multiple reflections in the crystal, this part is oscillating in $d$. Thus a measurement of $\Theta$ and $E$ as a function of $d$ can yield the intrinsic Faraday effect. The two perturbing terms can be rather large, an example gives the theoretical curve in fig. 4.

In our case the matter is more complicated. A guide acts as a medium with two different propagation constants for mutually perpendicular linearly polarized waves. Consequently the transmission and reflection at a crystal - wave guide interface depends on the degree of rotation and ellipticity of the incident wave, propagated through the rotating medium. In the coupler the wave incident upon the primary surface is always linearly polarized, so the transmission is constant. As for the second surface it must be borne in mind that $\Theta$ and $E$ are deduced from two measurements of phase and amplitude, the first with the wave guides parallel, the second with the wave guides in the crossed position. Now it can be shown that in the results of these two measurements the secondary guide acts as an isotropic, virtual, medium. In this medium circularly polarized waves can propagate, so the splitting up of $\Theta$ and $E$ into the three parts remains possible. The multiple reflection factor $M$ is, however, extremely complicated and can by no means be written in the simple form as given above. It remains a factor oscillating in $d$. Especially the difference in the phase angles of the reflection coefficients of the semiconductor - free space and the semiconductor - wave guide interfaces is large. Consequently it can be expected that the phase of the observed multiple reflection term, taken as a function of $d$, differs widely from the calculated one for the free space case.

3. Calculation of specific rotation and ellipticity. The rotation $\Theta$ and the ellipticity $E$ were calculated for $n$-type germanium, oriented with the (001) direction parallel to the external magnetic field. In this orientation the crystal behaves as if it were isotropic. A linearly polarized wave can be. split up into two circularly polarized ones, having different propagation constants ${ }^{7}$. With the simplifying assumption of an energy independent, isotropic, relaxation time, and using the well-known four ellipsoid model for $n$-type germanium, the conductivity tensor is exactly given by:

$$
\begin{aligned}
\sigma_{x x} & =\sigma_{y y}=\frac{1}{4} n e\left[\frac{4}{3}(2 K+1) \mu_{/ /}(1-i \omega \tau) \cdot \Gamma\right] \\
\sigma_{x y} & =-\sigma_{y x}=\frac{1}{4} n e\left[\frac{4}{3} K(K+2) \mu_{1 /}^{2} \cdot B \cdot \Gamma\right] \\
\Gamma & =\frac{1}{(1-i \omega \tau)^{2}+\frac{1}{3} K(K+2) \mu_{/ 1}^{2} B^{2}}
\end{aligned}
$$

with $n=$ number of carriers per unit volume, $-e=$ electron charge, $K=m_{/ /} / m_{\perp}$, rate of longitudinal to transverse effective mass in an ellip- 
soid, $\mu_{/ /}=$longitudinal mobility $=e \tau / m_{/}, \omega / 2 \pi=$ applied frequency, $\tau=$ relaxation time, and $B=$ applied external magnetic field.

For any specimen $n \cdot e$ and $\mu_{\| l}$ can be deduced from measurements of the d.c. conductivity and Hall coefficient, following standard lines. $K$ can be obtained from cyclotron resonance data, assuming $K$ is temperature independent. This is for $n$-type germanium probably a good approximation ${ }^{12}$ ).

With:

$$
\mathfrak{U}=\omega \mu_{0}\left(\omega \varepsilon+i \sigma_{x x}\right), \mathfrak{B}=\omega \mu_{0}\left(i \sigma_{x y}\right)
$$

where $\mu_{0}=$ the permeability of free space and $\varepsilon=$ the dielectric constant, the propagation constants of right- and left handed circularly polarized fields are given by the equations:

$$
\gamma_{ \pm}^{2}=\mathfrak{A} \pm i \mathfrak{B} .
$$

If $\gamma_{ \pm}=\alpha_{ \pm}+i \beta_{ \pm}$, the specific rotation and ellipticity are given by the formulae

$$
\begin{aligned}
& \Theta / l=\frac{\alpha_{-}-\alpha_{+}}{2} \\
& E / l=\left(\frac{\mathrm{e}^{-\beta_{-} l}-\mathrm{e}^{-\beta_{+} l}}{\mathrm{e}^{-\beta_{-l}}+\mathrm{e}^{-\beta_{+} l}}\right) \frac{1}{l}=\frac{\tanh \cdot \frac{1}{2}\left(\beta_{+}-\beta_{-}\right) l}{l}
\end{aligned}
$$

$l=$ distance from the zero point in the medium.

4. Results. Measurement on $n$-type germanium, (001) oriented, conductivity about $8 \Omega^{-1} m^{-1}$. The rotation and ellipticity were measured at room temperature as functions of the thickness $d$ of the crystal, at 24.9 $\mathrm{GHz}$. Two crystals were used, cut from the same single crystal. Crystal 1 was grinded in steps of about $0.2 \mathrm{~mm}$ from $3.26 \mathrm{~mm}$ down to $0.93 \mathrm{~mm}$, crystal II from 5.08 to $2.95 \mathrm{~mm}$.

Rotation. The results are shown in fig. 2, for three magnitudes of the external magnetic field $B$. With crystal II some measurements were performed for other fields, for $d=5.08 \mathrm{~mm}$. The importance of multiple reflections is evident. The penetration depth $\delta$ of the E.M. field is $3 \mathrm{~mm}$ in this case, it is clear that it is impractical to deduce the intrinsic effect from measured rotations for $d \leqslant \delta$. The rotation of crystal II was a lew percent higher than that of crystal I, for the same thicknesses. The supposition that the doping level was somewhat higher in the first case was confirmed by Hall measurements.

The form of the $\theta-d$ curve of crystal $\mathrm{I}$, for an applied magnetic field $B=0.63 \mathrm{~Wb} / \mathrm{m}^{2}$ was compared with the simplified theoretical one, calculated following the procedure given in paragraph 2 , i.e. for perpendicularly incident plane waves impinging on a semi infinite slab in free space. In this 
calculation the Hall mobility $\mu_{H}$ was assumed to be $\left.0.450 \mathrm{~m}^{2} / \mathrm{V} \cdot \mathrm{s}^{13}\right)$, and $K=m_{/ /} / m_{\perp}=19.3$. The conductivity $\sigma$ was found to be $\sigma=7.95 \Omega^{-1} m^{-1} \pm$ $2 \%$, at $23^{\circ} \mathrm{C}$, , using a two point method. The calculated intrinsic effect is given by the straight line in fig. 2 , the total rotation by the point-line curve.

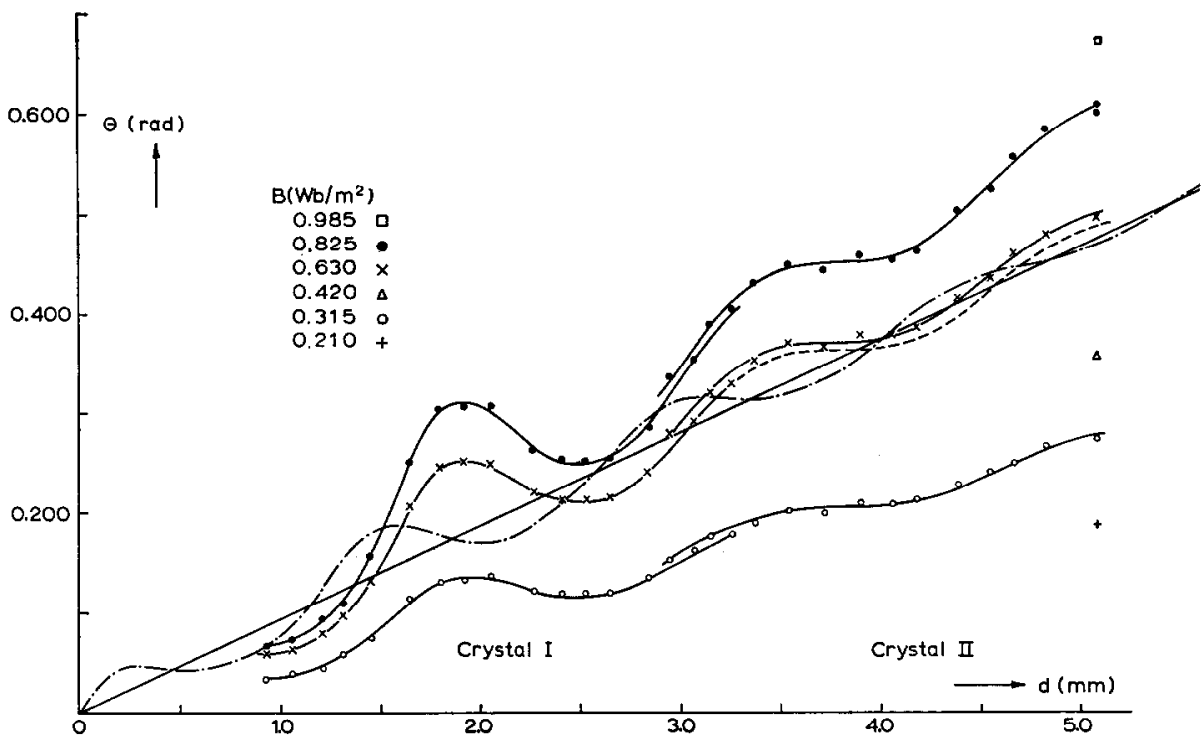

Fig. 2. Rotation $\Theta$ as a function of thickness $d$, for different magnetic fields $B$. Germanium, (001), $\sigma=8 \Omega^{-1} \mathrm{~m}^{-1}$. Calculated curves for $B=0.63 \mathrm{~Wb} / \mathrm{m}^{2}$ and $\mu_{H}=0.45$ $\mathrm{m}^{2} /$ V.s: straight solid line: intrinsic rotation, $-\cdot-\cdot-$ : total rotation for the free space case. ---- : extrapolated experimental results.

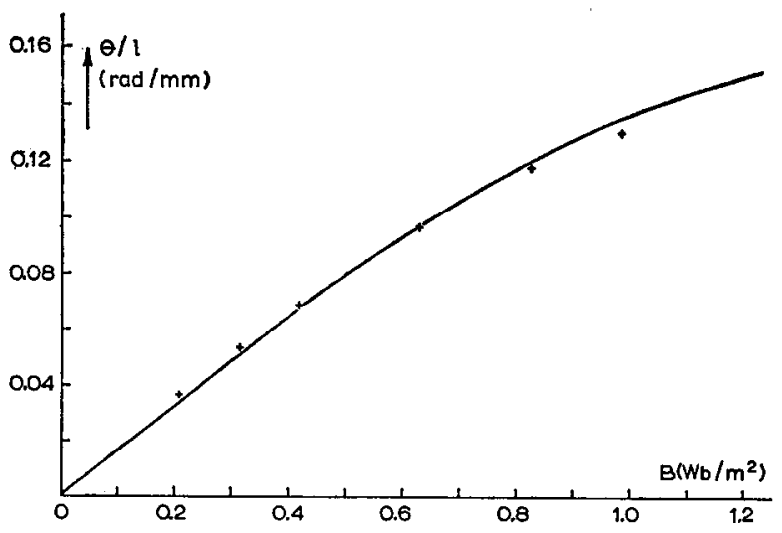

Fig. 3. Specific rotation as a function of magnetic field $B$. Germanium, (001), $\sigma=$ $8 \Omega^{-1} \mathrm{~m}^{-1}$. + : experimentally observed values, _- : calculated values, assuming $\mu_{H}=$ $0.45 \mathrm{~m}^{2} /$ V.s. 
The experimental curve was extrapolated from $3 \mathrm{~mm}$ upwards. For higher $d$ values the theoretical amplitude of the undulation is of the same order of magnitude as the measured one. The phase, however, is different, as can be expected. (see paragraph 2).

From the graphs in fig. 2 the specific rotation as a function of $B$ was derived. (fig. 3). For a good comparison with the theory we checked the Hall mobility of the sample, using the method described by van der $\mathrm{Pauw}{ }^{14}$ ). The result was $\mu_{H}=0.42 \mathrm{~m}^{2} / \mathrm{V} \cdot \mathrm{s} \pm 4 \%$, assuming there is no mixed conduction in the crystal. In fact there is a small amount of mixed conduction in the crystals used. However, the Hall mobility and the Faraday rotation are influenced in about the same magnitude by it. In view of the simplified model used in the calculations (constant isotropic relaxation time) the agreement between theory and experiment is surprisingly good, i.e. within a few percent. For higher $B$ values there is some divergence.

Ellipticity. At the same time as the rotation the ellipticity $E$ was measured as a function of $d$. The ellipticity as a function of the conductivity $\sigma$ passes through zero ${ }^{3}$. For $n$-type germanium, (001) oricntcd and at room temperature, this zero occurs for $\sigma=4.5 \Omega^{-1} \mathrm{~m}^{-1}$. For $\sigma=8 \Omega^{-1} \mathrm{~m}^{-1}$ the value of $E$ is still very small, but increases rapidly with increasing $\sigma$. Measuring these small $E$ values is revealing with respect to the sensitivity that can be obtained.

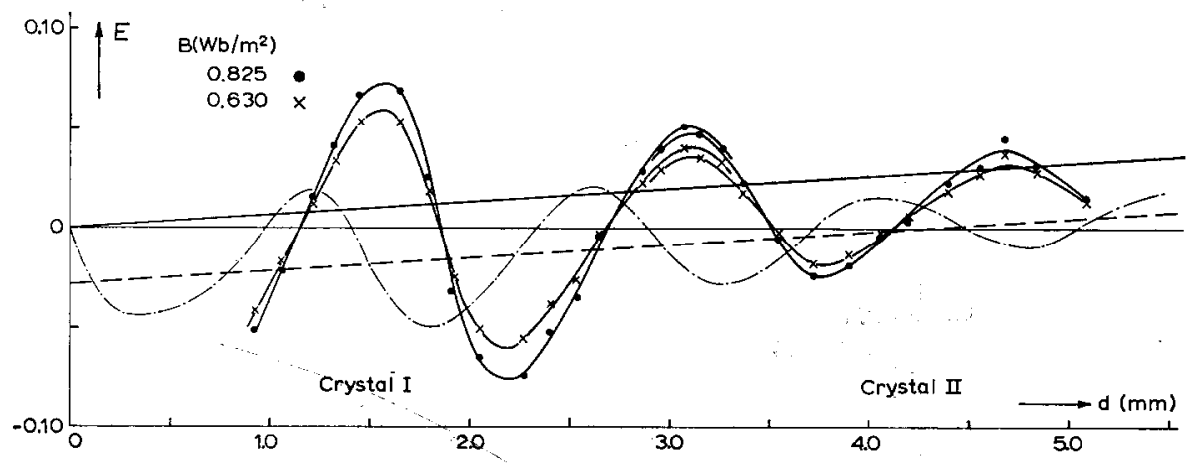

Fig. 4. Ellipticity $E$ as a function of thickness $d$. Germanium, (001), $\sigma=8 \Omega^{-1} \mathrm{~m}^{-1}$. Straight solid line: calculated intrinsic ellipticity, for $B=0.63 \mathrm{~Wb} / \mathrm{m}^{2},---$ : cal- $^{2}$ culated effect including surface effects, for the free space case, $-\cdot-\cdot-$ : calculated total ellipticity, for the free space case.

In fig. 4 the experimentally obtained $E$, as a function of $d$, for $B=0.63$ $\mathrm{Wb} / \mathrm{m}^{2}$, is compared with the theoretically expected one, calculated for crystal I. The two perturbing effects are clearly demonstrated, both experimentally and theoretically. For low $d$ values the measured $E$ can be many times as large as the intrinsic $E$, and opposite in sign. Using the curve of crystal II we estimated the intrinsic ellipticity to be, for $d=5.0$ 
mm.: $E=0.030 \pm 0.015$. This was theoretically expected to be 0.035 . Again no useful information can be deduced from measurements for $d$ lower than the penetration depth, as was the case with the rotation.

In order to make sure that the agreement between theory and experiment is more than accidental, measurements on another $n$-type germanium crystal, having a higher conductivity, have been carried out.

Measurements on $n$-type germanium, (001) oriented, $\sigma=26.7$ $\Omega^{-1} \mathrm{~m}^{-1}$. In this case the penetration depth $\delta$ of the E.M. field is about $1 \mathrm{~mm}$. The results as for the rotation are shown in fig. 5 . When $d$ exceeds $2 \delta$ the multiple reflection part of $\Theta$ is below the experimental error. The perturbing surface rotation, however, is clearly present, the magnitude is about $10 \%$ of the intrinsic rotation for $d=2 \mathrm{~mm}$. From the graphs the

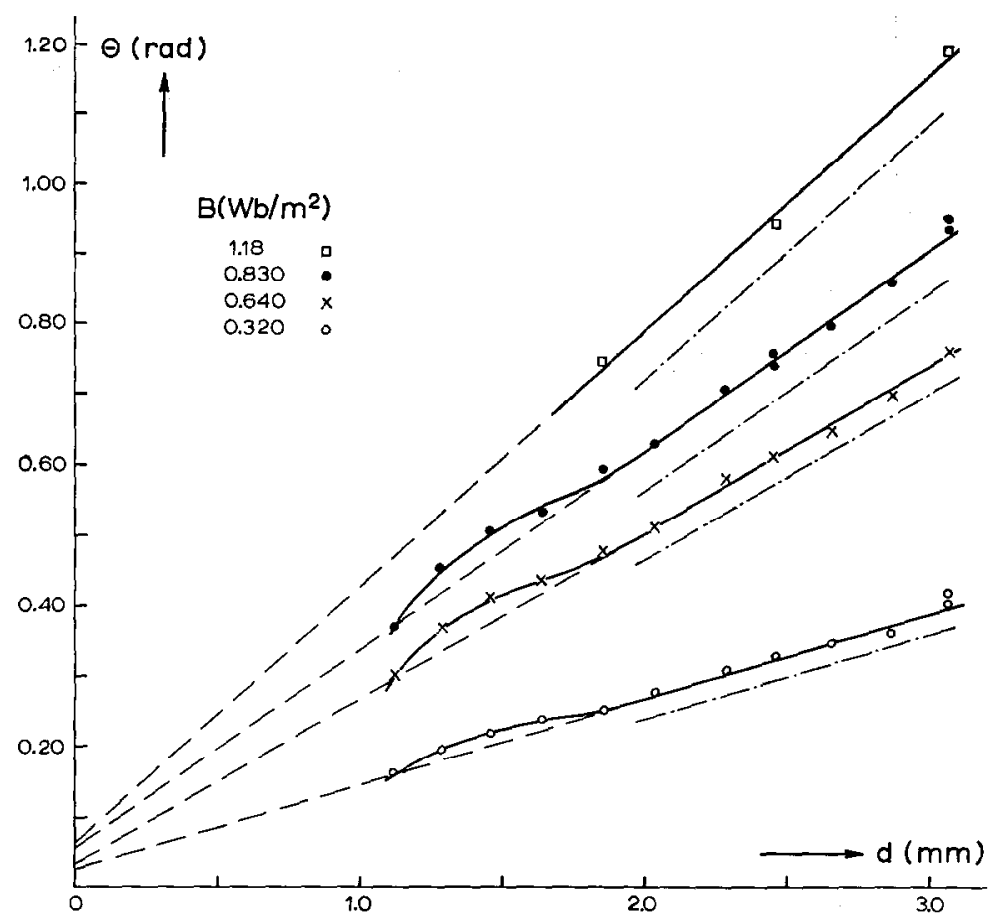

Fig. 5. Rotation $\Theta$ versus thickness $d$, for different $B$. Germanium, (001), $\sigma=27 \Omega^{-1} \mathrm{~m}^{-1} . \quad-\cdot-\cdots \cdot-$ : intrinsic rotation.

specific rotation as a function of $B$ was deduced (fig. 6). The solid curves represent the calculated specific rotation, assuming $\mu_{H}=0.40$ and 0.45 $\mathrm{m}^{2} / \mathrm{V} \cdot \mathrm{s}$ respectively. The first assumption clearly gives the better fit. With d.c. Hall measurements we found $\mu_{H}=0.41 \mathrm{~m}^{2} / \mathrm{V} \cdot \mathrm{s} \pm 4 \%$. In this case mixed conduction can be ignored. Thus the agreement between theory and experiment is still better than in the case of lower conductivity. Again 
some divergence for higher magnetic fields may be noticed (about $5 \%$ at $1.2 \mathrm{~Wb} / \mathrm{m}^{2}$.). Important is that the marked errors are mainly due to uncertainties in the magnitude of the surface effects.

In the same way the magnitude of $\frac{1}{2}\left(\beta_{+}-\beta_{-}\right)$as a function of $B$ was derived from measurements of the ellipticity as a function of $d$. In fig. 6

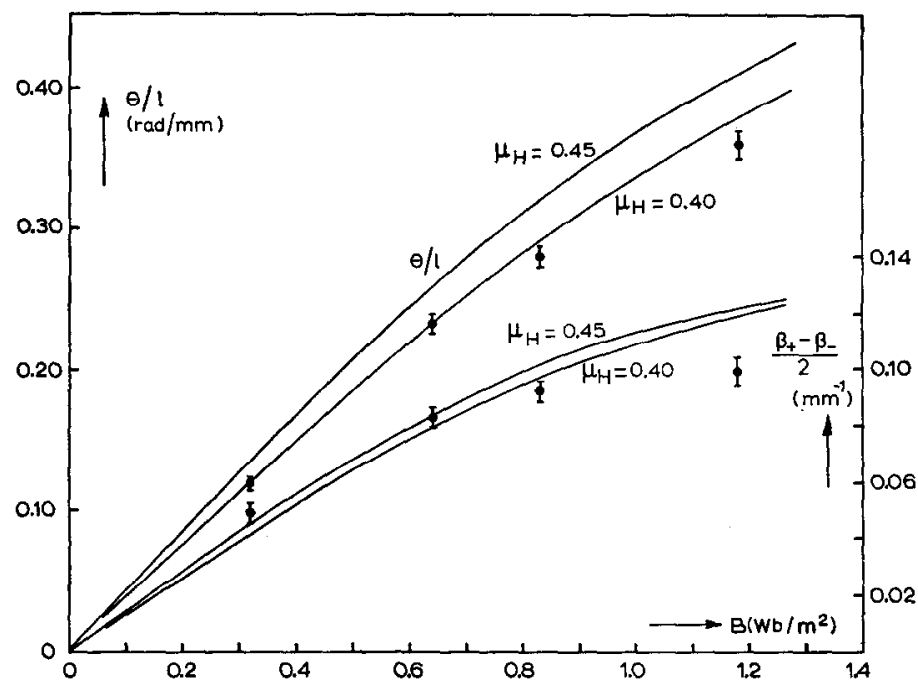

Fig. 6. Specific rotation and the function $\frac{1}{2}\left(\beta_{+}-\beta_{-}\right)$versus $B$. Germanium, (001), $\sigma=27 \Omega^{-1} \mathrm{~m}^{-1}$. Solid curves: calculated values, assuming $\mu_{H}=0.45$ and $0.40 \mathrm{~m}^{2} / \mathrm{V} . \mathrm{s}$ respectively.

the experimental data have been compared with the calculated ones, using again $\mu_{H}=0.40$ and $0.45 \mathrm{~m}^{2} / \mathrm{V} \cdot \mathrm{s}$. The marked errors are again a result of ambiguities in the interpretation.

5. Conclusion. With the crossed wave guide method one is able to measure the intrinsic Faraday effect at microwave frequencies with a fair degree of accuracy. The latter is mainly restricted by the occurrance of the perturbing effects of surfaces and multiple reflections in the measured sample. These effects can be eliminated in a simple way by measuring rotation and ellipticity as functions of thickness, assuming the latter is more than the penetration depth of the electromagnetic field. For the measurement of anisotropy effects at medium magnetic fields ${ }^{8}$ ) this method can be considered as very useful.

Acknowledgements. The authors are indebted to $\mathrm{mr}$. W. de Vos for his assistance in the early stage of this investigation, and to mr. M. H. van Maaren for his help with the d.c. Hall measurements. This investi- 
gation is part of the research program of the "Stichting voor Fundamenteel Onderzoek der Materie", and was made possible by financial support from the "Nederlandse Organisatie voor Zuiver Wetenschappelijk Onderzoek".

\section{Note added in proof.}

Recently, calculations of rotation and ellipticity have also been carried out with the assumption of an energy dependent, isotropic, relaxation time, characteristic for phonon scattering $\left(\tau \sim \varepsilon^{-\frac{1}{2}}\right)$. As for the rotation these calculations give an agreement between experiment and theory that lies within the experimental error, even for the highest fields, for the case given in fig. 6. As for the ellipticity the improvement is only a slight one. The conclusion remains, therefore, that the limitations set to the plane wave treatment, which is used in the interpretation of the experimental results, seem to be of little importance.

Received 6-4-63

\section{REFERENCES}

1) Suhl, H. and Pearson, G. C., Phys. Rev. 92 (1953) 858.

2) Rau, R. and Caspari, M. E., Phys. Rev. 100 (1955) 632.

3) Furdyna, J. K. and Broersma, S., Phys. Rev. 120 (1960) 1995.

4) Stephen, M. J. and Lidiard, A. B., J. Phys. Chem. Solids 9 (1958) 43.

5) Donovan, B. and Webster, J., Proc. Phys. Soc. 78 (1961) 120.

6) Furdyna, J. K. and Brodwin, M. E., Phys. Rev. 124 (1961) 740.

7) Donovan, B. and Webster, J., Proc. Phys. Soc. 79 (1962) 46.

8) Donovan, B. and Webster, J., Proc. Phys. Soc. 81 (1963) 90.

9) Champlin, K. S., Physica 28 (1962) 1143.

10) Hambleton, G. E. and Gärtner, W. W., J. Phys. Chem. Solids 8 (1958) 329. Proc. Symp. Millimetcr Waves, Ncw York, (1959) 87.

11) Schafer, G. E., IRE Transactions on Instrumentation I. 9 (1960) 217.

12) Bagguley, D. M. S., Stradling, R. A. and Whiting, J. S. S., Proc. Royal Soc. A262 (1961) 354.

13) Putley, E. H., The Hall effect and related phenomena ,Butterworths, (1960) 212.

14) Pauw, L. J. v. d., Phil. Res. Rep. 13 (1958) 1. 